\title{
Research on Optimization of Queuing System Based on Computer Simulation
}

\author{
Xiaosong Zhou \\ \{ zhouxiaosongwh@126.com\} \\ Department of Computer, Wuhan polytechnic Wuhan 430074, China
}

\begin{abstract}
This paper firstly introduces the queuing theory and discrete event system simulation of the basic knowledge, including queuing theory basic concept, the basic composition, research contents, performance metrics and several classical queueing system model, the discrete event system simulation steps. Then the characteristics, modeling principles and basic steps of several commonly used modeling methods are studied, mainly DFD diagram, IDEF0 diagram and Petri net, and the UML language based on the object-oriented thought. Then introduces the simulated annealing algorithm, tabu search algorithm and genetic algorithm, such as the characteristics of the heuristic algorithm, the basic idea, the main operation, and analysis and comparison. On the basis of the above theory, a general simulation model of queuing system is established by using UML modeling language and object-oriented analysis and design method. According to the characteristics of the optimization problem of the queuing system, an optimization strategy is proposed. Simulation optimization software was designed and developed. With appropriate service station opened number as the target of genetic algorithm optimization, queuing system simulation model based on the established, C + + language is used to write the simulation program, simulate the system operation, obtained with the system are closely related to the performance parameters of the customer queuing length and customer waiting time, compared with predefined metrics, as genetic algorithm of the adaptive function, and reproduction, crossover and mutation operation, after a certain number of iterations, finally obtained optimal solution.
\end{abstract}

Keywords: Queuing system; computer simulation; optimization; genetic algorithm; UML language

\section{Introduction}

In our daily life, we will encounter all kinds of queuing every day. For example, the bank teller to line up, the train station to buy tickets to line up, supermarkets, shopping malls in the payment to line up, booking hotels or tickets to line up, people seem to be in a line of society. However, with the accelerated pace of life, people cherish the time, more and more do not have the patience to long line. Therefore, in the face of increasingly fierce competition, as each consumer to complete the transaction must pass through the road - queuing system to a prominent position [1].

If the open window is too small, waiting for customers will be a very long time, the customer is not satisfied, which led to the loss of customers or to too many competitors when opening the window, while reducing the waiting time of a customer, but will cause idle waiter, 
the operating costs of enterprises increased. Therefore, how to according to the customer flow and the required payment time dynamic land, reasonable to set up toll window number, the customer satisfaction and enterprise operating costs can not be too high. This is an urgent problem. So the optimization problem of queuing system is produced. Queuing system optimization problem is divided into two types of system design optimization and system control optimization, the former is called static problem, which is called dynamic problem. In the given quality index to seek the optimal design of the problem, known as the optimization problem of the design of the queuing system, this kind of problem is generally easier to solve. Another type of system control optimization problem or dynamic problem, it is the function of time, it is much more difficult to solve than the former. The optimization problem of the system control is divided into the optimal control of the service equipment and the optimal control of the input process. The research focus of this paper is to optimize the service equipment in the control optimization of queuing system.

\section{Queuing Theory and Discrete Event Simulation System Construction Method}

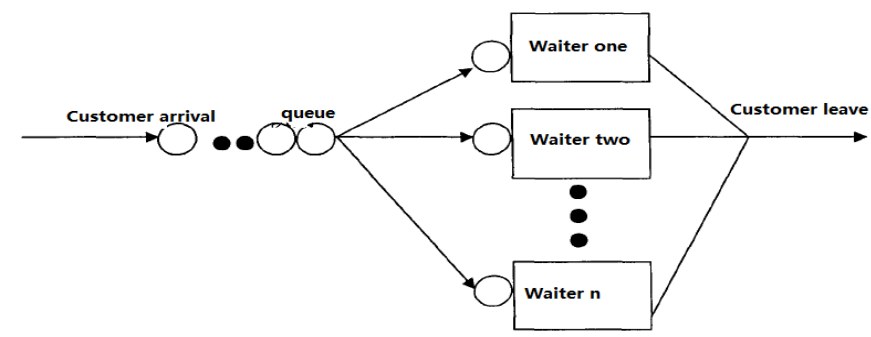

Fig. 1. Multi server queuing system

Queuing system consists of two aspects, one is asked to be served, the other to give service to the other side. We claim service person or thing (equipment) are collectively referred to as customer, give service providers or services referred to as waiters or service (sometimes waiter refers to the human, and the service station refers to give the service equipment). Customers and service stations form a queuing system, or a random service system. Obviously, the lack of customers and service stations are not formed on either side of the queuing system. Some of the queuing phenomenon in the form of visible, such as the work and other buses, such as the line we call visible. Some in the form of intangible, such as many customers call to order to order tickets, when which a customer is on the phone when other customers had to beside their telephone waiting, they may scattered in various places [2], but formed an invisible queue waiting for the call. The queuing phenomenon known as invisible line. 


\subsection{Research Problems in Queuing}

Randomness is one of the common characteristics of all kinds of queueing systems and plays a fundamental role. Queuing theory is mainly divided into three parts

(1) The problem of the nature of the queuing system

Queuing system of state of various queueing performance index of the system, including the captain of the system the system service desk of the number of customers, customer waiting time and the length of stay, and busy period etc.. The problem of queueing system is the core of queuing theory, and is the basis of the statistical inference and optimization of queuing system.

(2) The statistical inference of queuing system

In order to grasp a queuing system is running in the law, you need to by several observations, data collection, and using mathematical statistics method to get data processing, inferred by observing the queuing system, so that it can be used relevant theory to study and solve the problems related to the queuing system. The statistical inference of the queuing system is the basic work of the theoretical results. It is very necessary to develop the statistical inference method of the special stochastic processes, which is based on the basic work of the actual system [3].

(3) The optimization problem of queuing system

The optimization problem of the queuing system includes the optimal design of the system (static optimal) and the optimal operation control of the existing system (dynamic optimal). The former is in the service system settings, the future operation of the situation has been estimated, so that designers have a basis. The latter is to have the queuing system to seek the optimal operation strategy, it is the function of time, it is more difficult to solve than the former, complex. The key to speak for most practical system, if the input as determined by the objective conditions, uncontrolled (sometimes also can take the method to control the input), solve this problem is determine the combination of selected customer service rules or these amount or rate of services or service units, which is optimal in some sense.

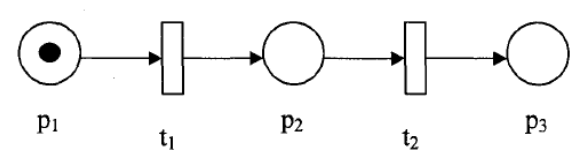

Fig. 2. The sequential execution of system modeling

\subsection{System Modeling Method}

In system science, a system is distinguished from other systems by its unique characterization and intrinsic characteristics, which are mainly composed of four aspects: the entity, attribute, activity and environment of the system. Entity is a specific object property system is to describe the characteristics of entities often to state and parameter characterization of the activity is changing with time change of the state of the environment, said system interface including interference and restriction.

From the modeling point of view, there are four aspects of the task for any system to study and describe. If the model is to be able to replace the actual system, the system must be able to reflect the characteristics of the system and the main characteristics of the system. Mathematical model is the basic condition for the analysis and control of dynamic systems by 
mathematical methods. The mathematical model is a useful and concise method to summarize the knowledge of the process, which is an effective tool for teaching and learning. Practice has two kinds of basic method can obtain the mathematical model of the system, one is theoretical method, namely use the system to follow the laws of physics are theoretical derivation, mechanism modeling on the other a experimental methods, namely, analysis experimental data, find out the relationship between the physical quantity, called system identification.

In view of the queuing system, there are two common methods, that is, the general description and simulation language description. General description method is the basic method of queuing system modeling, and its essence is to describe the basic elements of the system, which is the content of this section. And the simulation language description method, it uses the program language automatic processing to complete the statistical operation of the queuing system. For the queuing system, it has two basic operations, team operation and operation team. The exact form of the queuing model will depend on the number of servers and the number of queues. The most basic model is single queue, single server equipment system, more complex system can be modified by this model, enhanced to be described [4].

\subsection{Petri net Modeling Method}

Petri net is a kind of combined model which can be expressed by graph, which has the advantages of being intuitive, easy to understand and easy to use. In addition, Petri nets are strictly defined mathematical objects, with the help of mathematical analysis methods and techniques developed by Petri network can be used for static structural analysis, more can be used for dynamic behavior analysis. The goal of the Petri network is not to add a new member to a large number of system models. The rich and profound theory of Petri network makes it different from any other system model, its development will surely lay a solid theoretical foundation for information theory. The application fields of Petri nets cover all fields of computer science, such as circuit design, network protocol, software engineering, artificial intelligence, formal language, operating system, parallel compiling and data management, etc.. And the combination of object-oriented technology and multimedia also has great potential. At the same time, it is a powerful tool to study discrete event dynamic system, and the two successful application fields are performance evaluation and communication protocol.

$t_{1}$

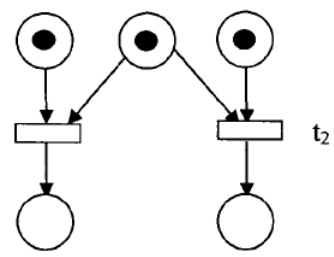

$t_{1}$

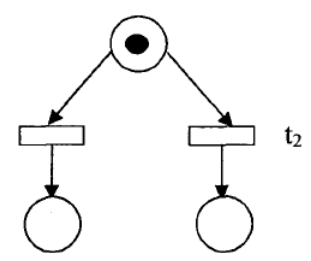


Fig. 3. Schematic diagram of the execution conflict of Petri network

\section{Genetic Algorithm}

The genetic algorithm is based on the "survival of the fittest" principle, optimization algorithm has a highly parallel, random and adaptive characteristics. It will be problem is expressed as a "chromosome" survival of the fittest process, through "chromosome" group of evolving from generation to generation, including reproduction, crossover and mutation, and eventually converge to the "best adapted to environment of the individual, so as to obtain the optimal solution or satisfactory solution. The coding technique and genetic operation of genetic algorithm are relatively simple, and the optimization of the genetic algorithm is not restricted by the restrictive conditions. The two most prominent features of genetic algorithm are implicit parallelism and global solution space search. Genetic manipulation is random in the whole process of evolution, but it is not completely random. It can effectively use historical information to predict the performance of the next generation of expected performance to be improved. This generation to continue to evolve, finally converge to a most adapt to the environment of the individual, optimal solution of the problem. Genetic algorithm involves five major elements include parameter coding, initial population setting, optimal design and control parameters of the value function design, genetic operation set [5].

\section{Conclusion}

This paper proposes to use UML to model the queuing system, and establishes a general model of queuing system simulation based on UML. And the optimization method and simulation technology are combined to solve the optimization problem of queuing system, and a simulation model is established. The use of UML to establish queuing system simulation model, as the basis for the preparation of queuing system simulation program. When you run the program, the output under certain arrival and service conditions, open service units, the performance index of the system, and a preset index compared, as an optimization problem where the objective function. In this paper selected as the adaptive function of genetic algorithm. And then carry out genetic operations, and return to a new generation of population (the number of service units), once again run the simulation program, the above operation. This iteration, until the optimal solution to the optimization problem of the queuing system is obtained. The optimal number of the 9 service stations is opened.

\section{References}

[1] Zhu Ding, Martha, Ming Duan Tang, Zhang Xuefeng. Based on Tabu Search simulated annealing particle swarm optimization algorithm of firepower distribution system. Simulation journal. No. 09. (2015)

[2] 000. Junzhou. Traveling salesman problem simulated annealing algorithm for. software technology based on. No. 15. (2013)

[3] Liang Di, Xie Liyang, Donghai, still Wenli. Based on IDEF\&UML production process management system modeling. Mini micro computer systems. No. 05. (2006) 
[4] Guo Zhuanghui, Hu Ke. Typical technology and application of. System simulation technology based on Petri net model. No. 02. (2011)

[5] Gao Haichang, Feng Boqin, Zhu Li B. intelligent optimization algorithm for solving TSP problems. Control and decision making. No. 03. (2013) 